\title{
MEMS system for fire detection in the forest
}

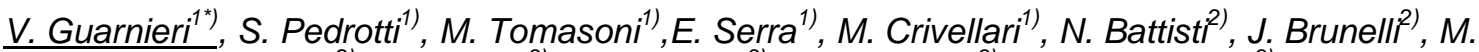 \\ Lorengo ${ }^{2)}$, A. Vasiliev $^{3)}$, A. Pisliakov ${ }^{3)}$, N.Samotaev ${ }^{3)}$, A. Varfolomeev ${ }^{3)}$ \\ ${ }^{1)}$ Fondazione Bruno Kessler, Via Sommarive 18, 38123 Trento, Italy \\ ${ }^{2)}$ Optoelettronica Italia S.r.l., Via Vienna 8, 38121 Gardolo (TN), Italy \\ ${ }^{3)}$ National research center "Kurchatov Institute", Kurchatov sq., 1, 123182, Moscow, Russia, \\ Corresponding e-mail address:guarni@fbk.eu
}

\begin{abstract}
This work focuses on the implementation of a MEMS fire detection system for controlling wide remote forest regions. The system is based on an innovative approach exploiting MEMS sensors with specific functions for detecting gas, wind and temperature, integrating data collected by a custom software. The aim of the work is to detect variations of the parameters measured by the sensors because any variation will promptly identify the presence of a forest fire. The technology and the structure of the system will be used also for other different applications of environmental monitoring, both outdoor and indoor.
\end{abstract}

Keywords: MEMS, gas, wind and temperature sensors, fire detection, environmental monitoring

\section{Introduction}

Recent advancement of technology and new challenges related with requirements to improve the safety and security of human life make necessary the production of novel instruments used for the early detection of threats confronting people in everyday activity. One of the most important problem is the development of new reliable devices for early detection of fire, in particular, early detection in forests, especially in dry Mediterranean climatic area and in thinly populated forest area of Russian Federation. The problem of early fire detection can particularly be solved by application of gas fire detectors (GFD), which sense the smell of fire long before the appearance of smoke or flame, or smoldering - at the moment when critical overheating begins. The production of these detectors became possible now due to the recent development of a new generation of sensitive and selective gas sensors characterized by extremely low power consumption and sensitivity. The most important target gases, which should be detected by such detectors are hydrogen and carbon monoxide: these gases are emitted in the very initial stage of fire, in the pyrolisis of combustible material accompanying the smoldering.

Fire Monitoring Integrated System described in this paper regards the development of an instrument to prevent and monitor forest fires. It is based on a wireless low energy consumption network, that consists of several nodes and is supplied by solar panels. Every node should be equipped with innovative sensors, monitoring different environmental parameters: from the noise of radiation and gases, which are emitted during the fire initial phase, to the wind speed direction and the temperature in order to prevent the spreading. Output signals of different sensors are electronically treated and wirelessly communicated to a central station that is able to determinate fire danger level though complex software.

\section{Gas sensor}

$\mathrm{CO}, \mathrm{H}_{2}$ gases are directly related to pyrolysis in forest fires. As a smell gases appear at lower temperatures than smoke, therefore gas fire detectors capable to identify them, can give alarm signal while open fires and even smouldering has not developed yet.

The new sensor is designated for this. The originality of the technology is related to the selective determination of fire gases (first of all $\mathrm{CO}$ and $\mathrm{H}_{2}$ ) in the mixture and possibility to measure different gases at once using temperature programmed measurement cycle. Moreover, unlike conventional optical smoke detectors, gas detectors are less influenced by dust particles or water vapour.

New gas sensors are based on micro hot plates over silicon oxide/silicon nitride layers and realized by microfabrication technology 
satisfying requirements like minimum power consumption, small dimensions, low cost and long-term stability (Fig.1A and 1B). Silicon oxide and silicon nitride layers were deposited by CVD technique.

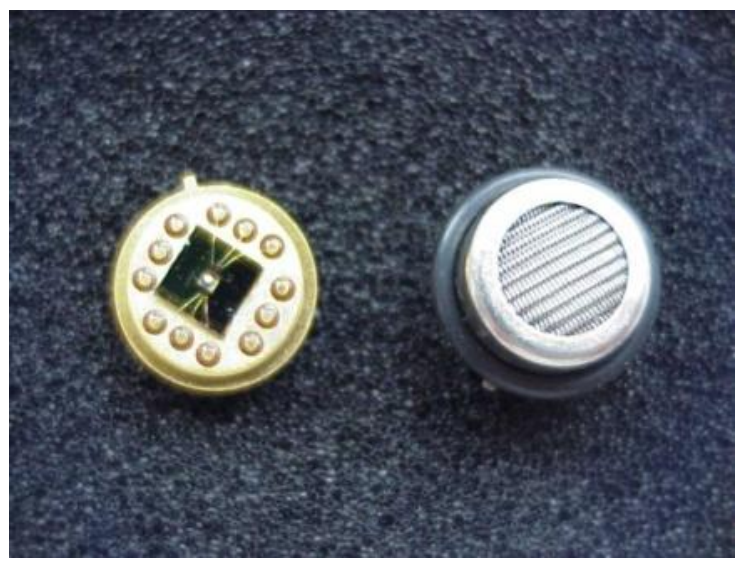

Fig. 1. A. Packaged gas sensors fabricated by using the technology "nano-on-micro"; it is gas sensing nano-material ( $P d$-doped Tin dioxide with particle size of about $10 \mathrm{~nm}$ ) deposited on MEMS substrate.

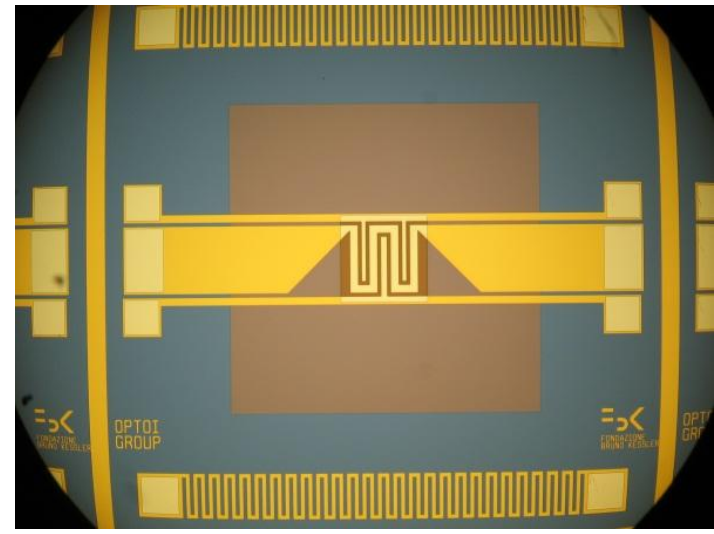

Fig. 1. B. Optical microscope photograph (5X magnification) of a gas sensor chip with thin multilayer dielectric membrane, Pt heater, and interdigitated electrodes.

Platinum microheater, used to reach the working temperature, maintains as high a film temperature as $450^{\circ} \mathrm{C}$ with less than $50 \mathrm{~mW}$ of input power. It was fabricated by sputtering of the metal and lift-off photo-litography. Lower temperature for our target gases and a pulse mode heating system allows decreasing the power consumption up to $1 \mathrm{~mW}$.

Sensing layer consists of $3 w t . \% \mathrm{Pd}$ doped $\mathrm{SnO}_{2}$ nanopowder with specific area of about 100 $\mathrm{m}^{2} / \mathrm{g}$. The sensing material was prepared by direct precipitation of Tin dioxide powder from a solution of Tin (II) sulphate using oxidation with hydrogen peroxide solution. This powder was carefully washed in centrifuge using deionised water, precipitated, dried at $600^{\circ} \mathrm{C}$, mixed with a solution of ethylcellulose in terpineol to get printable ink, and then deposited by screen printing over dielectric membrane with heater. Finally, the chip was annealed at $\sim 750^{\circ} \mathrm{C}$ to get desired sensitivity to target gases.

Detection principle of the gas sensor, which can be used for simultaneous detection of $\mathrm{CO}$ and hydrogen was described in [1]. Palladium is deposited on the surface of tin dioxide particles as clusters. This clusters are oxidised in air at temperature of $450^{\circ} \mathrm{C}$ and form p-type semiconductor material $\mathrm{PdO}$. The contact between p-type $\mathrm{PdO}$ with n-type $\mathrm{SnO}_{2}$ leads to the depletion of tin dioxide particles. At lower temperature, $\mathrm{PdO}$ can interact with $\mathrm{CO}$ (starting from $\sim 80^{\circ} \mathrm{C}$ ) and with hydrogen (starting from $\sim 150^{\circ} \mathrm{C}$ ). This interaction leads to the reduction of $\mathrm{PdO}$ and to increase in electron concentration (and conductivity) of $\mathrm{SnO}_{2}$.

Results of selective measurement of $\mathrm{CO}$ and $\mathrm{H}_{2}$ are presented in Fig. $2 \mathrm{~A}$ and $2 \mathrm{~B}$. The measuring cycle consists in the heating of the sensing layer up to $450^{\circ} \mathrm{C}$ for $0.5 \mathrm{~s}$ necessary for the oxidation of $\mathrm{Pd}$ to $\mathrm{PdO}$. Heating is followed by cooling the sensing layer to $110^{\circ} \mathrm{C}$ for the detection of $\mathrm{CO}$ and to $250^{\circ} \mathrm{C}$ for the detection of $\mathrm{H}_{2}$. At $110^{\circ} \mathrm{C} \mathrm{PdO}$ can oxidise $\mathrm{CO}$ to $\mathrm{CO}_{2}$ (see the drift in Fig. 2B), but the oxidation of $\mathrm{H}_{2}$ is much slower (drift part in Fig. 2A). At $250^{\circ} \mathrm{C}$, the oxidation of $\mathrm{H}_{2}$ with $\mathrm{PdO}$ is faster than the oxidation of $\mathrm{CO}$.

Therefore, the application of microcontroller and of the appropriate program enable the selective measurement of both gases with the same sensor operating alternatively in two regimes: $450-110-450-250-450^{\circ} \mathrm{C}$. Thus, the duration of whole period necessary for the detection of both gases is equal to $6 \mathrm{~s}$.

\section{Wind and temperature sensor}

For the prediction and localization of the source of fire, it is necessary to determine not only the concentration of fire indicating gases, but also the measure velocity and direction of wind responsible for the propagation of these gases. Therefore, the whole system must include also the sensors of air velocity.

The sensors are designed and fabricated using MEMS technology, the working principle is the measurement of the resistance of the hot platinum meander type anemometer placed on free-standing microbridge [2] with width of 200 $\mu \mathrm{m}$ and thickness of $1.1 \mu \mathrm{m}$ (Fig. $3 \mathrm{~A}$ and $3 \mathrm{~B}$ ).

Power consumption of the micro-heater is of around $10 \mathrm{~mW}$. Sheet resistance of platinum metallization is of $1.4 \mathrm{Ohm} / \mathrm{square}$ and TCR (Temperature Coefficient of Resistance) typically is of $2800 \mathrm{ppm} / \mathrm{C}$. Result of the air flow sensor is presented in Fig. 4. 


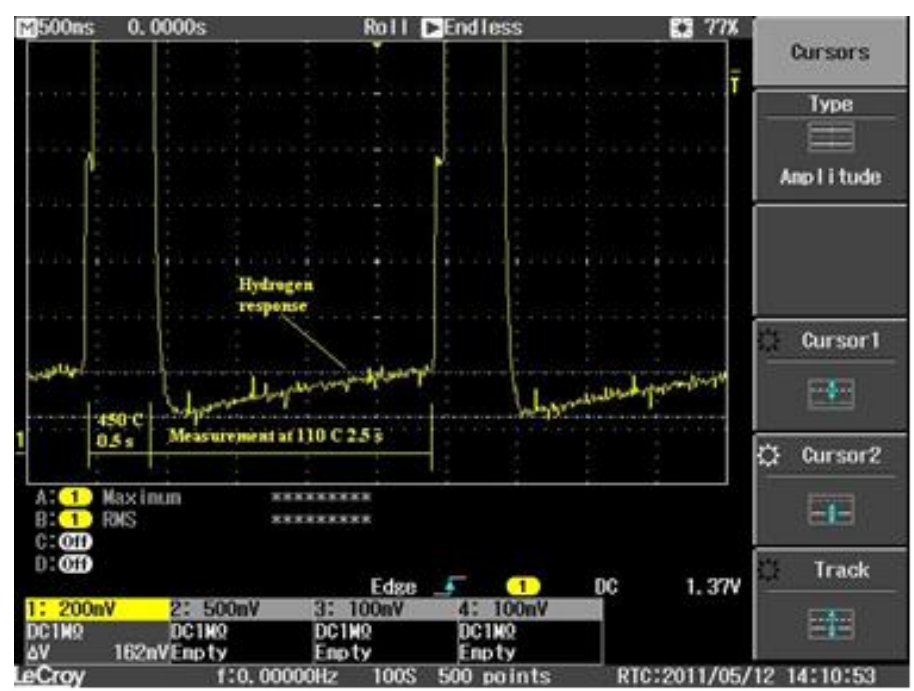

Fig. 2. A. Selective measurement of $\mathrm{CO}$ concentration compared to $\mathrm{H}_{2}$. Measurement cycle consists in the activation of the sensing layer at $450^{\circ} \mathrm{C}$ and the measurement of sensing layer conductivity drift at $110^{\circ} \mathrm{C}$. The slope of the curve is proportional to the CO concentration (3 ppm).

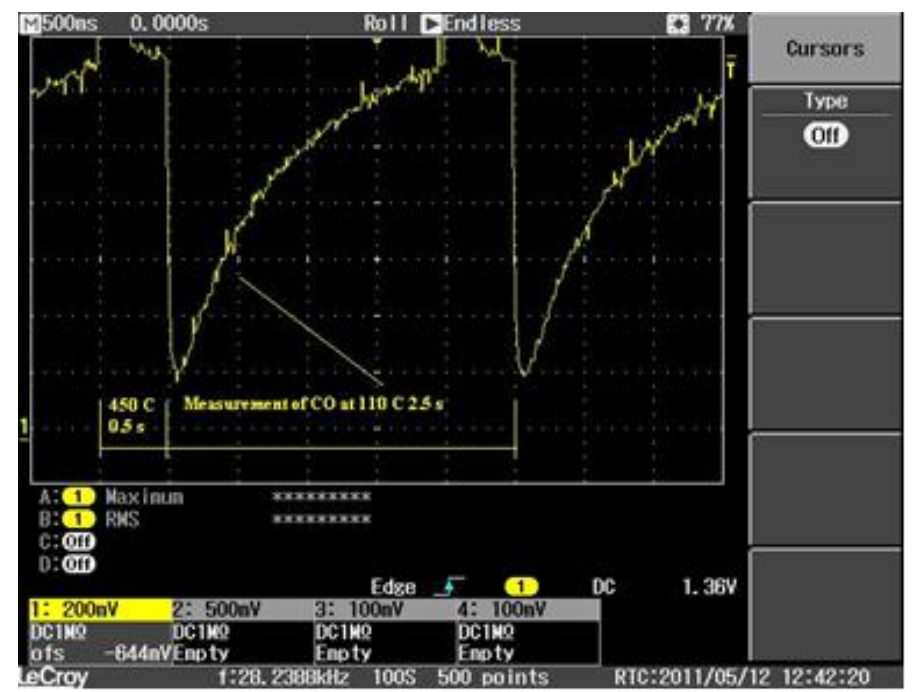

Fig. 2. B. Response

to $3 \mathrm{ppm}$ of $\mathrm{H} 2$ at the

same measurement

conditions of $\mathrm{CO}$.

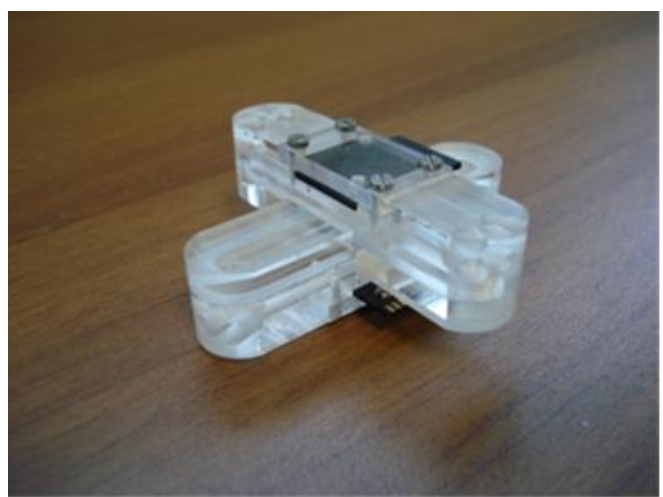

Fig. 3. A. Packaged Wind and temperature sensors used in the system for early detection of fire in forest.

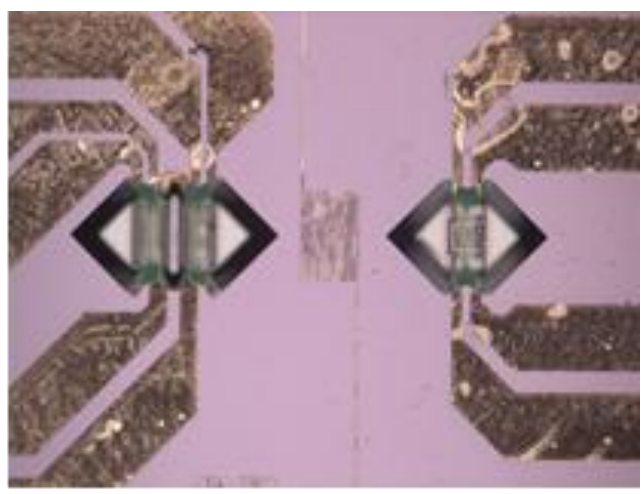

Fig. 3. B. Optical microscope photograph (5X magnification) of a wind and temperature MEMS die with thin multilayer dielectric suspended bridge, Pt heater and thermometer. 


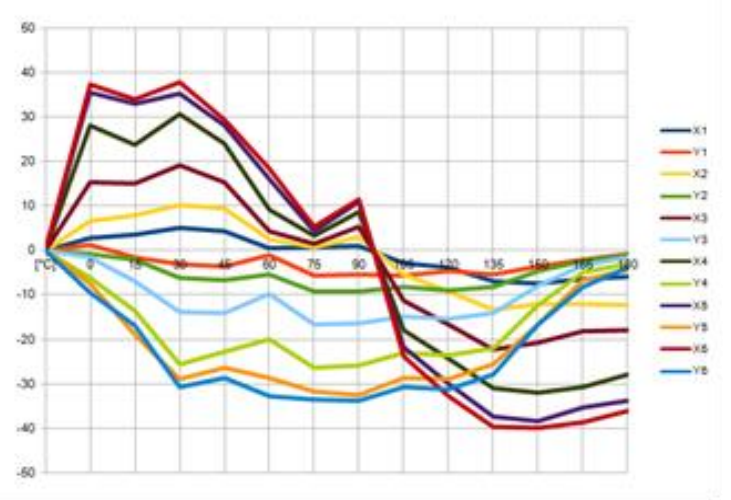

Fig. 4. Typical response of the air flow sensor $x 1=1 \mathrm{~m} / \mathrm{s}, x$ direction, $y 1=1 \mathrm{~m} / \mathrm{s}, y$ direction. wind and, using information of several sensors, to localize the source of fire.

Whole system containing gas sensors air flowmeters is controlled by ASIC, the scheme of this ASIC is presented in Fig. 5. The functions of this ASIC are the following: (1) Heater temperature control; (2) Bulk temperature monitoring; (3) Data acquisition and amplification; (4) Data offset correction; (5) Output signal linearization; (6) SPI communication with a microcontroller. ASIC is used also for the communication to a wireless low power node for data storage with SPI protocol.

Using two orthogonal air velocity sensors, it possible to detect both direction and velocity of

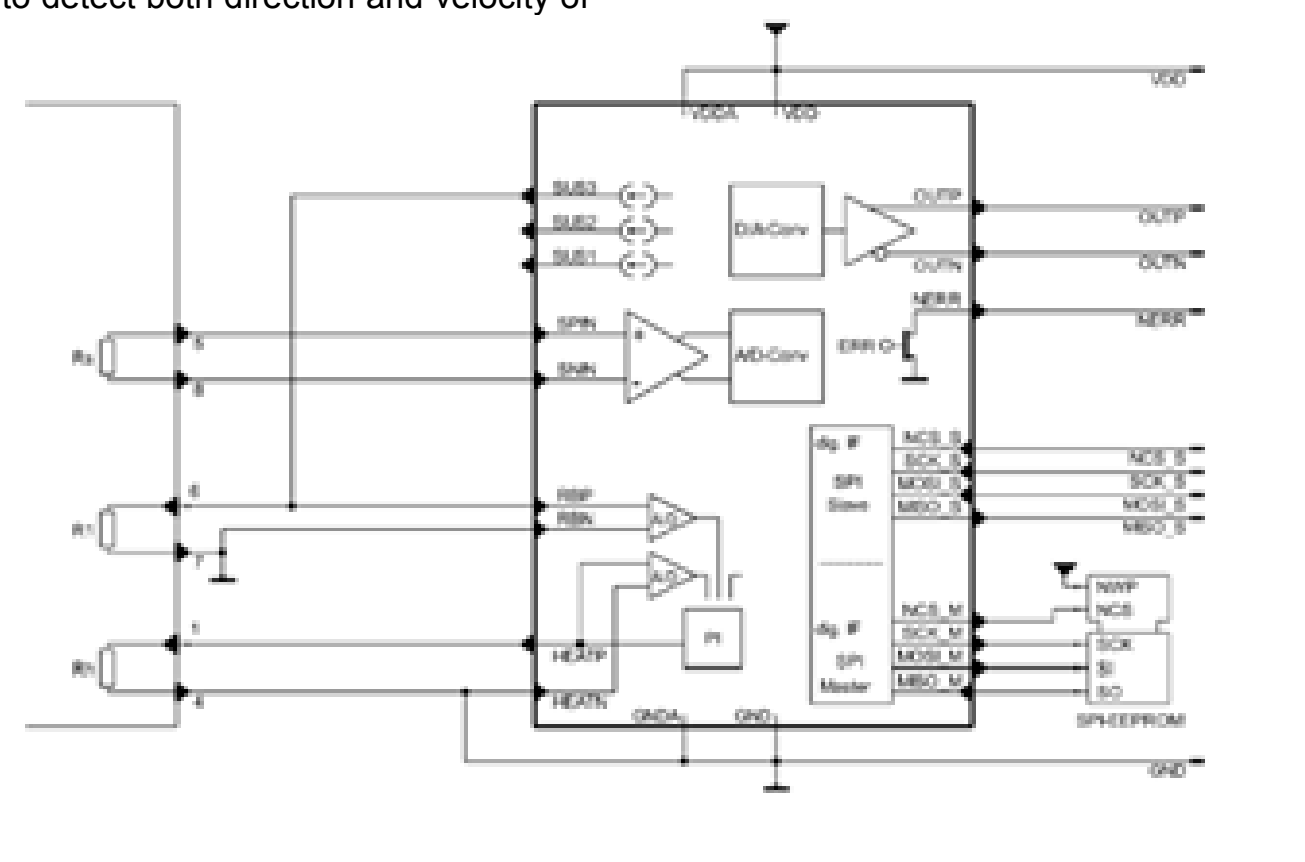

Fig. 5. The scheme of the ASIC used for the control of gas and air velocity sensors.

\section{Conclusion}

As a result of work, the system for early detection of starting fire in forest on the stage of smoldering of combustive materials is designed and fabricated. The detection limit of gas sensors of $\mathrm{CO}$ and $\mathrm{H}_{2}$ is shown to be of about 1 ppm. These gases can be detected selectively with a single sensor operating in pulsing heating regime. The field tests of the system showed its applicability for the localization of smoldering fires with area of about $1 \mathrm{~m}^{2}$.

\section{Acknowledgment}

This work has been funded by the "Legge provinciale 13 dicembre 1999, n. 6, articolo 5, PAT Provincia Autonoma di Trento" - FMIS (Fire Monitoring Integrated System) Project.

\section{References}

[1] N.N. Samotaev, A.A. Vasiliev, B.I. Podlepetski, A.V. Sokolov, A.V. Pislaikov. The mechanism of the formation of selective response of semiconductor gas sensor in mixture of $\mathrm{CH}_{4} / \mathrm{H}_{2} / \mathrm{CO}$ with air. Sensors and Actuators, B, 127, 242 - 247, 2007, doi: 10.1016/j.snb.2007.07.022

[2] Sekwang Park, Seunghyun Kim, Sunghyun Kim, Yongduk Kim. A flow direction sensor fabricating using MEMS technology and its simple interface circuit. Sensors and Actuators B 91 (2003) 347-352 doi: 10.1016/S0925-4005(03)00109-6 